\title{
Time Domain Harmonic State Estimation in Unbalanced Power Networks based on Optimal Number of Meters and the Principle of Half-Wave Symmetry
}

\author{
Ismael Molina-Moreno ${ }^{1}$, Aurelio Medina ${ }^{1 *}$, Rafael Cisneros-Magaña ${ }^{1}$, Olimpo Anaya-Lara ${ }^{2}$ \\ ${ }^{1}$ División de Estudios de Posgrado, Facultad de Ingeniería Eléctrica, Universidad Michoacana \\ de San Nicolás de Hidalgo, Av. Francisco J. Múgica S/N, Morelia, México \\ ${ }^{2}$ Department of Electronic and Electrical Engineering, University of Strathclyde, 204 George \\ Street, Glasgow, Scotland \\ *amedinr@gmail.com
}

\begin{abstract}
This paper proposes a time domain methodology for harmonic state estimation (HSE) in power systems. The proposed methodology is formulated to analyse the unbalanced operation of three-phase power systems including nonlinear loads. It takes into account the optimal number of measuring devices and exploits the property of half-wave symmetry in voltage and current waveforms to significantly reduce the computational effort of the solution process. The results obtained are in closely agreement with the actual response obtained from the time domain power system simulation performed with the SimPowerSystems toolbox of Simulink ${ }^{\circledR}$. Hence, the proposed methodology can adequately assess the harmonic state in unbalanced power systems. The results show a considerable reduction in the instrumentation resources and time processing needed for HSE in nonlinear power networks.
\end{abstract}

Keywords: Harmonics, power quality, state estimation, time domain, half-wave symmetry, Fourier analysis.

\section{Introduction}

Power quality is of important concern in the operation of the power systems [1]. Global power quality assessment has practical limitations due to the restricted number of monitored buses. To address this, power quality state estimation (PQSE) has been arisen [2-3]. Among the different variants of PQSE, the HSE will be explored in this research work.

HSE deals with a limited number of measuring devices to estimate the harmonic state at unmonitored buses in power systems [4-7]. Several contributions in the frequency domain have been reported to solve the HSE problem, e.g. the classic weighted least squares (WLS) [4-5], and the singular value decomposition (SVD) [6-7] have been used. However, all of them use voltage and current phasor quantities for each harmonic. This means that they should use power quality meters, which are quite expensive [8]. Besides, their adequate placement represents another challenging task as the number of measuring devices is limited. For this specific aim, several contributions have been reported [8-11].

The harmonic spectrum has conventionally been visualised by the magnitude and phase of each harmonic component, which can be determined through Fourier analysis, although there are other techniques successfully applied for this purpose [12-14]. An alternative way to represent the harmonic content is through a distorted signal waveform, i.e. harmonics are explicitly represented in the frequency domain and 
implicitly in the time domain [15]. Simulators such as the electromagnetic transient program (EMTP) [16] or Simulink ${ }^{\circledR}$ have been used to analyse power systems in the time domain. The most common meters to acquire voltage and current waveforms are through data acquisition (DAQ) systems. There are several contributions about DAQ systems, e.g. [17-18]. DAQ systems must be synchronised to correlate data acquired from multiple channels. DAQ systems manufacturers offer devices based on a time reference like GPS to ensure high performance synchronisation. The time skew on the clock waveforms is less than $1 \mathrm{~ns}$ [19].

The potential of the time domain approach is that the measurement process is less complex than in the frequency domain, as it is only necessary to take a snapshot of the waveform to capture all harmonic content, i.e. unlimited range of harmonics.

Reference [20] has addressed the time domain HSE (TDHSE) problem. The TDHSE method is based on Kalman filter (KF) which requires a priori knowledge of the harmonic sources, the error matrices associated with the measuring process, the model plant, and an initial state. However, it is not always possible to have knowledge of harmonic sources. The method uses the numerical differentiation method to obtain the periodic steady state solution of the power network [21] requiring of additional computational effort. The TDHSE solution uses an over-determined measurement state estimation equation.

Reference [22] proposes a method that exploits the property of half-wave symmetry in voltage and current waveforms. The method uses this feature to make more efficient the numerical differentiation method to obtain the periodic steady state solution of a power network, which is taken as the initial state to the Kalman filter method.

Reference [23] proposes a method based on Kalman filter as in [20] but the measurement equation is based on an under-determined condition. In addition, the method uses measurements taken from a scaledown test system set up, which allows observe the behaviour of the Kalman filter assessing the TDHSE when the theoretical model presents errors on modelling and parameter values respect to the real model.

Recently, in [24], a new methodology to solve the TDHSE problem using filtered measurements and the WLS method as criterion to minimise the estimation error has been proposed. In order to estimate the harmonic state, the method samples at least one cycle. The HSE formulation has been developed for singlephase networks.

The methodologies reported in [20] and [22-24] do not take into account the use of the optimal number of measuring devices and their optimal placement.

The proposed methodology extends the TDHSE formulation from single-phase [24] to three-phase networks to consider the unbalanced operation condition in power systems. It uses the half-wave symmetry 
property in voltage and current waveforms [22] to reduce from one to half cycle, the computational effort needed by the solution process of TDHSE. It introduces a procedure to obtain the minimum number of measuring devices to achieve full observability.

The rest of the paper is organised as follows: Section II describes the proposed TDHSE methodology divided into three main stages; Section III details the test systems for the conducted case studies; Section IV reports the results of the proposed methodology to evaluate the TDHSE through several conducted case studies; and Section V draws the main conclusions of this research work.

\section{TDHSE methodology}

The state estimation has four steps: 1) hypothesis structure, 2) state variables estimation, 3) error detection and 4) error identification and removal [25]. Hypothesis structure defines topology errors, bad data and parameter errors. The second step corresponds to the process of computing the state variables. Steps 3 and 4 verify the hypothesis model of step 1 and if necessary modify the model. Bad data is classified as extreme errors, gross errors and noise [3].

In this methodology, the hypothesis model considers no topology errors and no parameter errors; bad data only considers normal measurement noise. The proposed method is focused on step 2. Detection and identification of extreme and gross errors have been the purpose of other contributions, e.g. [26-27].

The complete scheme for the TDHSE solution is shown in Fig. 1. The measurement matrix is developed using a three-phase network to analyse the unbalanced operation of power systems. Since HSE deals with a limited number of measuring devices, the measuring process should take into account an optimal measuring system, which can be defined as a system with a minimum number of measuring devices to obtain full observability. Data may be corrupted by noise. A filter based on the fast Fourier transform (FFT) is applied for noise mitigation. To exploit the feature of symmetry, the voltage and current waveforms are sampled only during half-cycle. The TDHSE uses the measurement equation and the WLS criterion to minimise the estimation error. In order to change from time domain to frequency domain, the FFT is used. To apply FFT, the feature of half-wave symmetry is used to obtain an estimated complete cycle. 


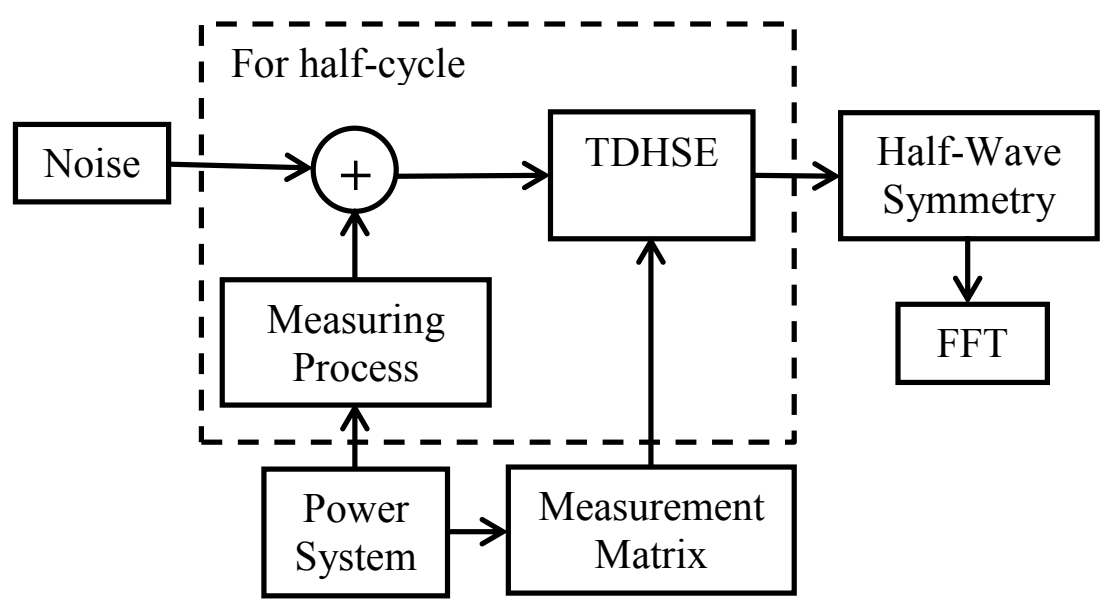

Fig. 1. Complete scheme for the TDHSE problem

\subsection{Measurement matrix}

TDHSE is based on the mathematical relationship between state variables and measurements, i.e.

$$
\mathbf{z}=\mathbf{H x}+\mathbf{e}
$$

where $\mathbf{x} \in \mathbb{R}^{n}$ is the state vector; $\mathbf{z} \in \mathbb{R}^{m}$ contains the measurements; $\mathbf{H} \in \mathbb{R}^{m \times n}$ is called measurement matrix and $\mathbf{e} \in \mathbb{R}^{m}$ represents the error associated to the measuring process generally assumed to be zero mean white Gaussian noise. The matrix $\mathbf{H}$ is said to be over-determined if $m>n$, under-determined if $m<$ $n$, or critically-determined if $m=n$ [28].

If the matrix $\mathbf{H}$ is over-determined, the WLS method is used to solve (1). It minimizes the sum of the squared deviations from actual measurements of estimated state [4-5]. The estimated vector $\hat{\mathbf{x}}$ is defined by:

$$
\widehat{\mathbf{x}}=\left(\mathbf{H H}^{\mathrm{T}}\right)^{-1} \mathbf{H z}
$$

If all measurements are critical, the loss of one of them means loss of observability. However, some measurements have more influence than others, namely leverage measurements. The over-determined condition can be used to support leverage measurements.

If the matrix $\mathbf{H}$ is under-determined, the SVD gives a solution using the pseudo-inverse [6-7], i.e. $\mathbf{H}$ can be factorised using SVD,

$$
\mathbf{H}=\mathbf{U S V}^{\mathrm{T}}
$$

where $\mathbf{S} \in \mathbb{R}^{n \times n}$ is a diagonal matrix with positive or zero elements called the singular values of $\mathbf{H}$. The columns of $\mathbf{U} \in \mathbb{R}^{m \times n}$ are the left singular vectors and the columns of $\mathbf{V} \in \mathbb{R}^{n \times n}$ are the right singular vectors. Then,

$$
\hat{\mathbf{X}}=\mathbf{V S} \mathbf{S}^{-1} \mathbf{U}^{\mathrm{T}} \mathbf{z}
$$

If the matrix $\mathbf{H}$ is critically-determined, 


$$
\widehat{\mathbf{x}}=\mathbf{H}^{-\mathbf{1}} \mathbf{z}
$$

To formulate the TDHSE problem, time $t$ is defined as,

$$
t[k]=k T_{\mathrm{S}}=t^{k}
$$

where $T_{\mathrm{S}}$ is called sample period and $k$ is the $k$-th sample number. The notation of $k T_{\mathrm{S}}$ will be represented by the super index $k$.

Numerical differentiation is needed to obtain $\mathbf{H}$. There are several formulas to obtain the derivative of a function [29]. The basic formulas are known as the forward-difference formula if $T_{\mathrm{S}}>0$ and the backward-difference formula if $T_{\mathrm{S}}<0$. These formulas generate an error $O\left(T_{\mathrm{S}}\right)$. In this research work, the three-point midpoint formula is used as it generates an error $O\left(T_{\mathrm{S}}^{2}\right)$. Hence, the derivative of the function $y$ at $t=k T$ is

$$
y_{(t=k T)}^{\prime}=\frac{y^{k+1}-y^{k-1}}{2 T_{\mathrm{S}}}+O\left(T_{\mathrm{S}}^{2}\right)
$$

The second derivative of the function $y$ at $t=k T$ can be obtained using the second derivative midpoint formula. This formula also generates an error $O\left(T_{\mathrm{S}}^{2}\right)$ and is defined as

$$
y_{(t=k T)}^{\prime \prime}=\frac{y^{k+1}-2 y^{k}+y^{k-1}}{T_{\mathrm{S}}^{2}}+O\left(T_{\mathrm{S}}^{2}\right)
$$

In [24], the elements more frequently analysed in power systems to form the measurement equation have been partially reported. The mathematical formulation is now extended to represent three-phase unbalanced power networks.

The proposed methodology is mainly formulated for transmission systems. However, since unbalance is a more usual operation condition in distribution systems, the methodology has been extended to represent, as a particular case, unbalanced distribution systems.

2.1.1 Transmission line modelled by an equivalent $\pi$-model: Fig. 2 shows a scheme for two buses, $s$ and $r$, connected by a transmission line. The transmission line is usually modelled by a distributed parameters model, but it can be represented by an equivalent $\pi$-model as there is only interest in the behaviour at input and output ends of the line. The transmission line model includes the following parameters: the self and mutual resistances, inductances, capacitances, and admittances, i.e. $\mathbf{R}, \mathbf{L}, \mathbf{C}$, and $\mathbf{G} \in \mathbb{R}^{3 \times 3}$, respectively, required to model the equivalent $\pi$-model [30]. 


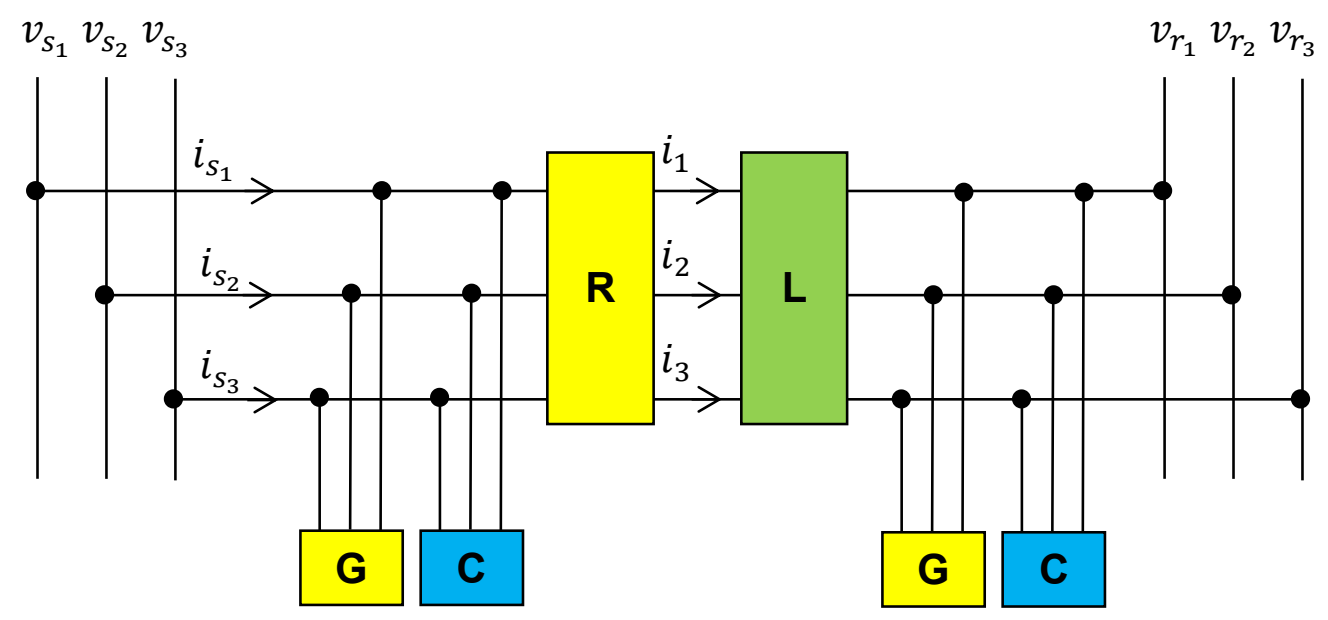

Fig. 2. Transmission line represented by an equivalent $\pi$-model

The series branch current, $\mathbf{i} \in \mathbb{R}^{3 \times 3}$, and the shunt currents, $\mathbf{i}_{G}$ and $\mathbf{i}_{C} \in \mathbb{R}^{3 \times 3}$ are not possible measurements as they are not physically available. However, by applying circuit theory in the continuoustime, the bus voltages $\mathbf{v}_{s}$ and $\mathbf{v}_{r} \in \mathbb{R}^{3 \times 3}$, can be related as,

$$
\mathbf{v}_{S}-\mathbf{v}_{r}=\mathbf{R i}+\mathbf{L i}^{\prime}
$$

where $\mathbf{v}_{s}=\left[\begin{array}{l}v_{s_{1}} \\ v_{s_{2}} \\ v_{s_{3}}\end{array}\right], \quad \mathbf{v}_{r}=\left[\begin{array}{l}v_{r_{1}} \\ v_{r_{2}} \\ v_{r_{3}}\end{array}\right], \quad \mathbf{R}=\left[\begin{array}{lll}R_{11} & R_{12} & R_{13} \\ R_{21} & R_{22} & R_{23} \\ R_{31} & R_{32} & R_{33}\end{array}\right], \quad \mathbf{i}=\left[\begin{array}{l}i_{1} \\ i_{2} \\ i_{3}\end{array}\right], \quad \mathbf{L}=\left[\begin{array}{lll}L_{11} & L_{12} & L_{13} \\ L_{21} & L_{22} & L_{23} \\ L_{31} & L_{32} & L_{33}\end{array}\right]$ and $\mathbf{i}^{\prime}=\left[\begin{array}{l}i_{1}^{\prime} \\ i_{2}^{\prime} \\ i_{3}^{\prime}\end{array}\right]$.

Applying the Kirchhoff current law (KCL) at bus $s$, the sending end current $\mathbf{i}_{s}$ is:

$$
\mathbf{i}_{s}=\mathbf{i}+\mathbf{i}_{\mathrm{G}}+\mathbf{i}_{\mathrm{C}}
$$

where $\mathbf{i}_{s}=\left[\begin{array}{l}i_{s_{1}} \\ i_{s_{2}} \\ i_{s_{3}}\end{array}\right], \quad \mathbf{i}_{\mathrm{G}}=\left[\begin{array}{l}i_{\mathrm{G}_{1}} \\ i_{\mathrm{G}_{2}} \\ i_{\mathrm{G}_{3}}\end{array}\right]$ and, $\mathbf{i}_{\mathrm{C}}=\left[\begin{array}{c}i_{\mathrm{C}_{1}} \\ i_{\mathrm{C}_{2}} \\ i_{\mathrm{C}_{3}}\end{array}\right]$.

Applying circuit theory, the continuous-time current $\mathbf{i}_{C}$ in the capacitor with parameter $\mathbf{C}$, placed at bus $s$, is:

$$
\mathbf{i}_{\mathrm{C}}=\mathbf{C} \mathbf{v}_{S}^{\prime}
$$

where $\mathbf{C}=\left[\begin{array}{lll}C_{11} & C_{12} & C_{13} \\ C_{21} & C_{22} & C_{23} \\ C_{31} & C_{32} & C_{33}\end{array}\right]$, and, $\mathbf{v}_{s}^{\prime}=\left[\begin{array}{c}v_{s_{1}}^{\prime} \\ v_{s_{2}}^{\prime} \\ v_{s_{3}}^{\prime}\end{array}\right]$

Applying the Ohm's law, the continuous-time current in an admittance $\mathbf{G}$ connected at bus $s$ is given by:

$$
\mathbf{i}_{\mathrm{G}}=\mathbf{G} \mathbf{v}_{s}
$$


where $\mathbf{G}=\left[\begin{array}{lll}G_{11} & G_{12} & G_{13} \\ G_{21} & G_{22} & G_{23} \\ G_{31} & G_{32} & G_{33}\end{array}\right]$

Hence, replacing (11) and (12) in (10) and solving for $\mathbf{i}$ gives,

$$
\mathbf{i}=\mathbf{i}_{S}-\mathbf{G v}_{S}-\mathbf{C} \mathbf{v}_{S}^{\prime}
$$

Please notice (13) is now left as a function of available measurements. The derivative of (13) is

$$
\mathbf{i}^{\prime}=\mathbf{i}_{s}^{\prime}-\mathbf{G} \mathbf{v}_{s}^{\prime}-\mathbf{C} \mathbf{v}_{s}^{\prime \prime}
$$

Substitution of (13) and (14) in (9) gives,

$$
(\mathbf{I}+\mathbf{R G}) \mathbf{v}_{s}+(\mathbf{R C}+\mathbf{L G}) \mathbf{v}_{s}^{\prime}+\mathbf{L C} \mathbf{v}_{s}^{\prime \prime}-\mathbf{v}_{r}=\mathbf{R} \mathbf{i}_{s}+\mathbf{L} \mathbf{i}_{s}^{\prime}
$$

where $\mathbf{I} \in \mathbb{R}^{3 \times 3}$ is the identity matrix [31].

To obtain the discretised measurement equation it is necessary to consider the samples $k$, the previous $k-1$, and the subsequent $k+1$. By applying (7) and (8) to discretise (15) leads to,

$$
(\mathbf{I}+\mathbf{R G}) \mathbf{v}_{S}^{k}+\frac{(\mathbf{R C}+\mathbf{L G})\left(\mathbf{v}_{S}^{k+1}-\mathbf{v}_{S}^{k-1}\right)}{2 T_{\mathrm{S}}}+\frac{\mathbf{L C}\left(v_{S}^{k+1}-2 \mathbf{v}_{S}^{k}+\mathbf{v}_{S}^{k-1}\right)}{T_{S}^{2}}-\mathbf{v}_{r}^{k}=\mathbf{R} \mathbf{i}_{S}^{k}+\frac{\mathbf{L}\left(\mathbf{i}_{S}^{k+1}-\mathbf{i}_{S}^{k-1}\right)}{2 T_{S}}
$$

Reordering (16) gives,

$$
\mathbf{c} \mathbf{1}_{s-r} \mathbf{v}_{s}^{k}+\mathbf{c} 2_{s-r} \mathbf{v}_{s}^{k+1}+\mathbf{c} 3_{s-r} \mathbf{v}_{s}^{k-1}-\mathbf{v}_{r}^{k}=\mathbf{b} \mathbf{1}_{s} \mathbf{i}_{s}^{k}+\mathbf{b} 2_{s} \mathbf{i}_{s}^{k+1}+\mathbf{b} \mathbf{3}_{s} \mathbf{i}_{s}^{k-1}
$$

where $\quad \mathbf{c}_{1, S-r}=\mathbf{I}+\mathbf{R G}-\frac{2 \mathbf{L C}}{T_{\mathrm{S}}^{2}}, \quad \mathbf{c}_{2, S-r}=\frac{\mathbf{R C}}{2 T_{\mathrm{S}}}+\frac{\mathbf{L G}}{2 T_{\mathrm{S}}}+\frac{\mathbf{L C}}{T_{\mathrm{S}}^{2}}, \quad \mathbf{c}_{3, S-r}=\frac{\mathbf{L C}}{T_{\mathrm{S}}^{2}}-\frac{\mathbf{R C}}{2 T_{\mathrm{S}}}-\frac{\mathbf{L G}}{2 T_{\mathrm{S}}}, \quad \mathbf{b}_{1, \mathrm{~S}}=\mathbf{R}, \quad \mathbf{b}_{2, S}=$ $\mathbf{L} / 2 T_{\mathrm{S}}$, and $\mathbf{b}_{3, S}=-\mathbf{L} / 2 T_{\mathrm{S}}$. The sub-index $s-r$ of the coefficients: $\mathbf{c 1}, \mathbf{c 2}$, and $\mathbf{c 3}$ is associated with the sending end and receiving end of the transmission line. The sub-index $s$ of the coefficients: b1, b2, and $\mathbf{b 3}$ is associated with the sending end bus voltage.

If the sending end bus $s$ is the instrumented bus of the transmission line, the measurements are the sending end voltage $\mathbf{v}_{\mathrm{zs}}$ and the sending end current $\mathbf{i}_{\mathrm{zs}}$. This condition gives a critically-determined measurement equation where,

$$
\mathbf{z}=\left[\begin{array}{c}
\mathbf{b} \mathbf{1}_{\mathrm{s}} \mathbf{i}_{\mathrm{zs}}^{k}+\mathbf{b} \mathbf{2}_{s} \mathbf{i}_{\mathrm{zs}}^{k+1}+\mathbf{b} \mathbf{3}_{s} \mathbf{i}_{\mathrm{z} s}^{k-1} \\
\mathbf{v}_{\mathrm{z} s}^{k-1} \\
\mathbf{v}_{\mathrm{z} s}^{k+1} \\
\mathbf{v}_{\mathrm{z} s}^{k-1}
\end{array}\right], \mathbf{H}=\left[\begin{array}{cccc}
\mathbf{c} \mathbf{1}_{s-r} & \mathbf{c} \mathbf{2}_{s-r} & \mathbf{c} \mathbf{3}_{s-r} & -\mathbf{I} \\
\mathbf{I} & \mathbf{0} & \mathbf{0} & \mathbf{0} \\
\mathbf{0} & \mathbf{I} & \mathbf{0} & \mathbf{0} \\
\mathbf{0} & \mathbf{0} & \mathbf{I} & \mathbf{0}
\end{array}\right] \text {, and } \quad \mathbf{x}=\left[\begin{array}{c}
\mathbf{v}_{s}^{k} \\
\mathbf{v}_{s}^{k+1} \\
\mathbf{v}_{s}^{k-1} \\
\mathbf{v}_{r}^{k}
\end{array}\right]
$$

Please notice that matrices $\mathbf{I}$ and $\mathbf{0} \in \mathbb{R}^{3 \times 3}$ are the identity and zero matrices, respectively.

2.1.2 Distribution line: For this type of line, the capacitance $\mathbf{C}$ and the admittance $\mathbf{G}$ are omitted. Hence, the number of coefficients in (17) is reduced as follows:

$\mathbf{c 1} \mathbf{1}_{s-r}=\mathbf{I}, \quad \mathbf{c} \mathbf{2}_{s-r}=\mathbf{0}, \quad \mathbf{c} \mathbf{3}_{s-r}=\mathbf{0}, \quad \mathbf{b} \mathbf{1}_{s}=\mathbf{R}, \quad \mathbf{b} \mathbf{2}_{s}=\mathbf{L} / 2 T_{\mathrm{S}}$, and $\quad \mathbf{b} \mathbf{3}_{s}=-\mathbf{L} / 2 T_{\mathrm{S}}$.

Rewriting (17) with the reduced coefficients gives, 


$$
\mathbf{v}_{s}^{k}-\mathbf{v}_{r}^{k}=\mathbf{b} \mathbf{1}_{s} \mathbf{i}_{s}^{k}+\mathbf{b} \mathbf{2}_{s} \mathbf{i}_{s}^{k+1}+\mathbf{b} \mathbf{3}_{s} \mathbf{i}_{s}^{k-1}
$$

Distribution systems are modelled with circuits of three or four wires. For four wire circuits, a fourth current $i_{0}^{k}$ called neutral current is defined as follows,

$$
i_{0}^{k}=i_{L_{1}}^{k}+i_{L_{2}}^{k}+i_{L_{3}}^{k}
$$

where $i_{L_{1}}^{k}, i_{L_{2}}^{k}, i_{L_{3}}^{k}$ are the current in phases 1,2 , and 3 , respectively.

2.1.3 Current in a load connected at bus $s$ : If the load is modelled by a resistance $\mathbf{R}_{\mathrm{L}}$ connected in parallel to an inductance $\mathbf{L}_{\mathrm{L}}$ supplied by $\mathbf{v}_{s}$, and $\mathbf{i}_{\mathrm{L}}$ is the current in the load, the equation that relates the current in the load and the supplying voltage is,

$$
\mathbf{i}_{\mathrm{L}}^{\prime}=\frac{\mathbf{v}_{S}^{\prime}}{\mathbf{R}_{\mathrm{L}}}+\frac{\mathbf{v}_{S}}{\mathbf{L}_{\mathrm{L}}}
$$

By applying (7) to discretise (20) gives,

$$
\mathbf{c} \mathbf{4}_{S} \mathbf{v}_{S}^{k}+\mathbf{c} 5_{s} \mathbf{v}_{S}^{k+1}+\mathbf{c} \mathbf{6}_{s} \mathbf{v}_{S}^{k-1}=\mathbf{i}_{\mathrm{L}}^{k+1}-\mathbf{i}_{\mathrm{L}}^{k-1}
$$

where $\mathbf{c} \mathbf{4}_{s}=2 T_{\mathrm{S}} / \mathbf{L}_{\mathrm{L}}, \quad \mathbf{c} \mathbf{5}_{s}=1 / \mathbf{R}_{\mathrm{L}}, \quad \mathbf{c} \boldsymbol{6}_{s}=-1 / \mathbf{R}_{\mathrm{L}}[32]$.

2.1.4 Current in a capacitors bank connected at bus s: The current in a capacitors bank with parameter $\mathbf{C}_{s}$ placed at bus $s$ can be obtained using (11). By applying (7) to discretise (11),

$$
\mathbf{v}_{s}^{k+1}-\mathbf{v}_{s}^{k-1}=\mathbf{b} \mathbf{4}_{s} \mathbf{i}_{C_{S}}^{k}
$$

where $\mathbf{b} \mathbf{4}_{s}=2 T_{\mathrm{S}} / \mathbf{C}_{s}$ and $\mathbf{i}_{C_{S}}$ is the current in the capacitors bank. Please notice that current in the capacitors bank can be measured [33].

2.1.5 Line current when bus voltages are known: In the frequency domain, if all bus voltages are known, the total network can be determined. In the time domain, this premise is also true. This is the reason to take the bus voltages as state variables. The series current in a transmission line of Fig. 2 can be determined solving (10) for $\mathbf{i}$ using a numerical method such as the Euler method, i.e.

$$
\mathbf{i}^{k}=\left(\mathbf{R}+\frac{\mathbf{L}}{T_{\mathrm{S}}}\right)^{-1}\left(\mathbf{v}_{S}^{k}-\mathbf{v}_{r}^{k}+\frac{\mathbf{L}}{T_{\mathrm{S}}} \mathbf{i}^{k-1}\right)
$$

Since $\mathbf{i}^{k-1}$ is not known, it can be initialized to zero and apply the numerical integration for a few cycles, to obtain a better initial approximation [3]. The shunt current in a transmission line can be also determined by discretising (11) and (12), i.e.

$$
\begin{gathered}
\mathbf{i}_{C}^{k}=\frac{\mathbf{C}}{2 T_{\mathrm{S}}}\left(\mathbf{v}_{S}^{k+1}-\mathbf{v}_{S}^{k-1}\right) \\
\mathbf{i}_{G}^{k}=\mathbf{G}_{S}^{k}
\end{gathered}
$$


Finally, the sending end current can be determined by the discretised form of (10), i.e.

$$
\mathbf{i}_{S}^{k}=\mathbf{i}^{k}+\mathbf{i}_{C}^{k}+\mathbf{i}_{G}^{k}
$$

\subsection{Measuring process}

The measuring process consists in the capture of voltage and current waveforms. The measuring devices can be DAQ systems. The number of channels of DAQ systems is practically unlimited because they can be connected in cascade. Since HSE deals with a limited number of measuring devices, an algorithm to optimise this limited number must be used. Unfortunately, data may be corrupted by noise, making necessary the use of a filter for its mitigation. These two issues are described next.

2.2.1 Optimal number of measuring devices: Measuring devices used in HSE have been power quality meters. To optimise the number of them, several contributions have been reported [8-11]. Since the proposed methodology is formulated in the time domain, an optimisation algorithm must be suggested. The reported algorithms to obtain full observability in the frequency domain [34-40] can be exploited, extending the concept to the time domain. The procedures to analyse the observability are the numerical analysis [3435] and the topological analysis [35-36]. This approach can be summarised in the following rules [36-39]:

Rule 1: Installation of a measuring device in a given bus makes itself and other buses incident to that bus observable [36-40].

There are two additional rules that incorporate the concept of zero injection bus (ZIB) [35-39]. A ZIB is a bus that does not inject current into the system. These additional rules to obtain observability are as follows,

Rule 2: If only one bus is unobservable among a ZIB and its entire incident buses, the unobservable bus will also be identified as observable by applying the Kirchhoff's current law (KCL) at the ZIB. Currents in the time-domain can be computed using (23-26).

Rule 3: If the entire incident buses connected to an unobservable ZIB are observable, the ZIB can be observable applying KCL at the ZIB.

The mathematical formulation that satisfies the rule of observability number 1 can be formulated as a problem of Integer Linear Programming (ILP) as follows [40],

$$
\begin{aligned}
& \operatorname{Min} \sum_{\mathbf{i}=\mathbf{1}}^{\mathbf{n}} \mathbf{d}_{\mathbf{i}} \\
& \text { S.T. Ad } \geq \mathbf{b}
\end{aligned}
$$

Where $n$ is the number of buses and $d_{i}$ is a binary variable with entries are 


$$
d_{i}= \begin{cases}1, & \text { if bus } i \text { is instrumented } \\ 0, & \text { otherwise }\end{cases}
$$

The matrix $\mathrm{A}$ is formed using the line data of the bus, i.e.

$$
a_{i j}=\left\{\begin{array}{l}
1, \quad \text { if } i=j \\
1, \text { if } i \text { and } j \text { are adjacents } \\
0, \quad \text { otherwise }
\end{array}\right.
$$

The vector $\mathbf{d} \in \mathbb{R}^{n}$ contains each possibility of monitoring the $n$ buses, i.e. $\mathbf{d}=\left[d_{1} d_{2} \cdots d_{n}\right]^{\mathrm{T}}$.

The full observability is achieved when the vector $\mathbf{b} \in \mathbb{R}^{n}$ is equal to $\left[\begin{array}{llll}1 & 1 & \cdots & 1\end{array}\right]^{\mathrm{T}}$; it means that each bus is observable at least once.

The ILP method uses voltage and current measurements without conventional measurements. However, from conventional measurements (injections and power flows), zero injections can be used as pseudo-measurements. In order to exploit the ZIB pseudo-measurement, the original topological observability is modified as follows: The ZIB and one of the incident buses are merged. With the new topological observability, (27) is used to determine the instrumented buses.

2.2.2 Filter based on Fourier transform: Measurements are commonly contaminated by noise. Noise affects differently each harmonic owing to harmonics are of different magnitude. To select the interest harmonic, a filter based on FFT is used. A summary of the proposed filter in [24] is given below:

A function $y(t)$ with period $T$ can be represented with a Fourier series as,

$$
y\left(k T_{\mathrm{S}}\right)=\frac{1}{T} \sum_{n=0}^{N-1} \mathbf{C}_{\mathrm{F}}(n) \mathrm{e}^{j 2 \pi n k T_{\mathrm{S}} / T} \quad k=0,1, \cdots, N-1
$$

where $\mathbf{C}_{\mathrm{F}}$ represents the Fourier coefficients. If $\mathbf{Y}$ is a vector that recollects $N$ samples from $y(t)$ corresponding to one period, then, the Fourier coefficients can be determined by the discrete Fourier transform (DFT) as,

$$
\mathbf{C}_{\mathrm{F}}(n)=\sum_{k=0}^{N-1} \mathbf{Y}(k) \mathrm{e}^{j 2 \pi n k T_{\mathrm{S}} / T} \quad n=0,1, \cdots, N-1
$$

The FFT is an alternative algorithm to compute the DFT. This transform has less computational complexity.

Once $\mathbf{C}_{\mathrm{F}}$ is determined, the coefficients of interest are selected to reconstruct the waveforms; hence, the harmonic of interest can be selected. Preliminary results for the three-phase balanced case have been reported in [24].

\subsection{Half-wave symmetry}

In this methodology it is assumed that the power system is operating in steady state. Hence, distorted voltage waveforms are periodic. Symmetry of waveforms with respect to the origin of coordinates is called 
half-wave symmetry [41]. In a periodic waveform with half-wave symmetry, the inverted negative halfwaveform is identical to the positive half-waveform, but is shifted by half period or $\pi$ radians respect to the positive half-waveform. Half-wave symmetrical waveform only exhibit odd harmonics and its mean value is zero [42]. A periodic waveform $v(t)$ is half-wave symmetrical if it satisfies,

$$
v(t)=-v\left(t \pm \frac{1}{2} T\right) \forall t
$$

For instance, let $v(t)=\sin (\omega t)+0.15 \sin (3 \omega t)-0.1 \sin (5 \omega t)+0.05 \sin (7 \omega t)$ be a voltage function, which is shown in Fig. 3.

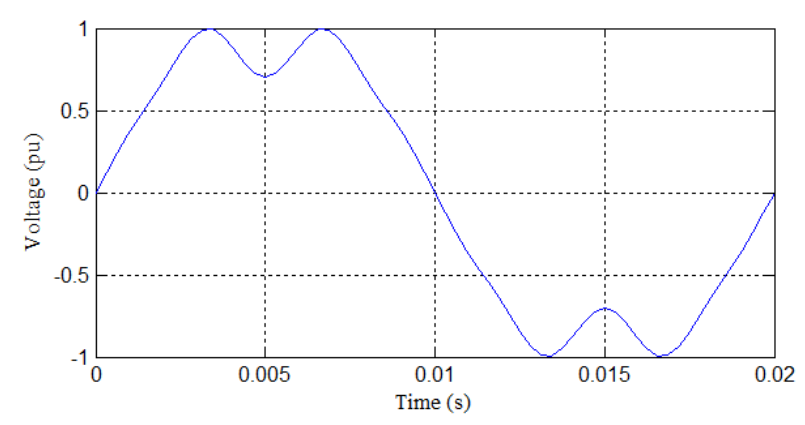

Fig. 3. Half-wave symmetrical waveform

To test the symmetry property, several values are sampled at times $k T$ and $k T+T / 2$; these values are given in Table 1 . They satisfy (30) to have the half-wave symmetry property.

Table 1 Half-wave symmetry property test

\begin{tabular}{cccc}
\hline$k T$ & $v(\mathrm{t})$ & $k T+T / 2$ & $v(\mathrm{t})$ \\
\hline 0.0000 & 0.0000 & 0.0100 & 0.0000 \\
0.0025 & 0.8485 & 0.0125 & -0.8485 \\
0.0050 & 0.7000 & 0.0150 & -0.7000 \\
0.0075 & 0.8485 & 0.0175 & -0.8485 \\
\hline
\end{tabular}

In order to exploit the half-wave symmetry property, the waveforms are sampled only for a half-cycle. If a cycle is divided into $N$ samples it is only necessary to process $N / 2$ samples. As a consequence, the time processing needed to estimate the HSE is reduced by approximately $50 \%$ as it only estimates half-cycle of the waveform.

\section{Test systems}


The modified Lower South Island of New Zealand test system is shown in Fig. 4(a). The test system data are reported in [16]. One bus has been instrumented to obtain a full observability, i.e. the criticallydetermined case. This condition is shown in Fig. 4(a). A three-phase harmonic current source is injected at bus T220. The harmonic current components are given in Table 2.

Table 2 Harmonic current injection

\begin{tabular}{|c|c|c|c|c|}
\hline \multirow[t]{2}{*}{ Harmonic } & \multicolumn{3}{|c|}{ Phase peak value (A) } & \multirow[t]{2}{*}{ Phase sequence } \\
\hline & A & $\mathrm{B}$ & $\mathrm{C}$ & \\
\hline 5 & 4.000 & 2.000 & 1.000 & - \\
\hline 7 & 2.000 & 1.000 & 0.500 & + \\
\hline 11 & 1.000 & 0.500 & 0.250 & - \\
\hline 13 & 0.500 & 0.250 & 0.125 & + \\
\hline
\end{tabular}

To evaluate the robustness of the proposed methodology, the modified IEEE 14-bus system, shown in Fig. 4(b) has been also selected as test system. Positive sequence data are taken from [43] while the zero sequence ones are given in Table 3. The measuring devices and their placement to obtain a full observability are shown in Fig. 4(b) as a green set. This condition corresponds to the determined case. According to the available measuring devices, more can be added to have redundancy and thus reduce the estimation error. For illustrative purposes, an additional set of measuring devices, marked as a red set, has been added to obtain an over-determined case. This set is also shown in Fig. 4(b) and identified as additional measurements.

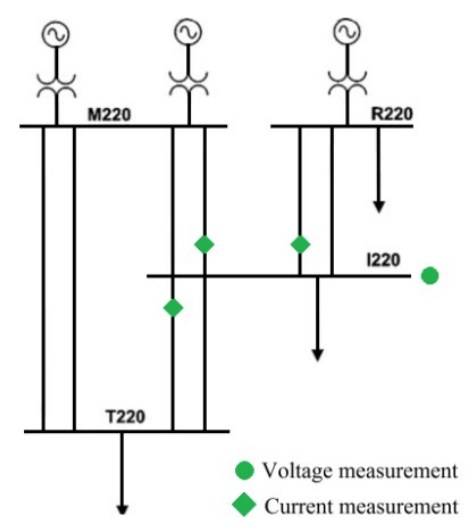

$a$ 


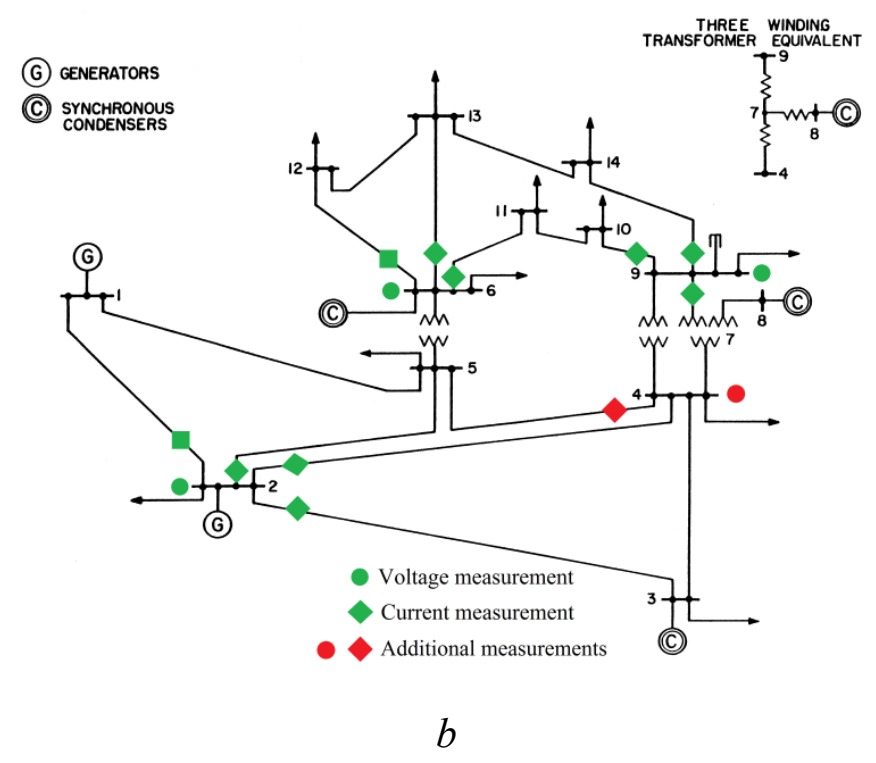

Fig. 4. Test systems

$a$ Modified Lower South Island of New Zealand

$b$ Modified IEEE 14-bus.

Table 3 Zero sequence parameter for modified 14-bus test system

\begin{tabular}{ccc}
\hline Line & $\mathrm{R}_{0}$ & $\mathrm{~L}_{0}$ \\
$1-2$ & 0.048450 & 0.147925 \\
$1-5$ & 0.135075 & 0.557600 \\
$2-3$ & 0.117475 & 0.494925 \\
$2-4$ & 0.145275 & 0.440800 \\
$2-5$ & 0.142375 & 0.434700 \\
$3-4$ & 0.167525 & 0.427575 \\
$4-5$ & 0.033375 & 0.105275 \\
$4-7$ & 0 & 0.209120 \\
$4-9$ & 0 & 0.556180 \\
$5-6$ & 0 & 0.252020 \\
$6-11$ & 0.237450 & 0.497250 \\
$6-12$ & 0.307275 & 0.639525 \\
$6-13$ & 0.165375 & 0.325675 \\
$7-8$ & 0 & 0.440375 \\
$7-9$ & 0 & 0.275025 \\
$9-10$ & 0.079525 & 0.211250 \\
$9-14$ & 0.317775 & 0.675950 \\
$10-11$ & 0.205125 & 0.480175 \\
$12-13$ & 0.552300 & 0.499700 \\
$13-14$ & 0.4227325 & 0.870050 \\
\hline & & \\
\hline
\end{tabular}


In order to add nonlinear loads, an AC-AC converter has been used. The AC-AC converter is controlled by the firing angle $\alpha$; hence, the load current is a nonlinear load connected to bus 4 . Reference [44] states that the output power $P_{\mathrm{o}}$ for a resistive load can be determined by,

$$
P_{\mathrm{o}}=\frac{V_{\mathrm{m}}^{2}}{2 R_{\mathrm{L}}}\left(1-\frac{\alpha}{\pi}+\frac{\sin 2 \alpha}{2 \pi}\right)
$$

where $V_{\mathrm{m}}$ is the maximum value of the voltage waveform, $R_{\mathrm{L}}$ is the resistive load and $\alpha$ the firing angle.

\section{Case studies}

\subsection{Case study 1: Comparative analysis of the proposed method}

The proposed methodology is compared against the Kalman filter and the Simulink ${ }^{\circledR}$ responses. The criteria to perform the comparison are the number of measuring devices used for the estimation and the accuracy of THD at unmonitored busbars.

The proposed methodology is performed in the test system shown in Fig. 4(a). There are $n=4$ buses which can be instrumented. According to (27), the resulting function to minimise is,

$$
\begin{gathered}
\operatorname{Min} \sum_{i=1}^{4} d_{i}=d_{1}+d_{2}+d_{3}+d_{4} \\
\text { S.T. }\left[\begin{array}{llll}
1 & 0 & 1 & 1 \\
0 & 1 & 1 & 0 \\
1 & 1 & 1 & 1 \\
1 & 0 & 1 & 1
\end{array}\right]\left[\begin{array}{l}
d_{1} \\
d_{2} \\
d_{3} \\
d_{4}
\end{array}\right] \geq\left[\begin{array}{l}
1 \\
1 \\
1 \\
1
\end{array}\right]
\end{gathered}
$$

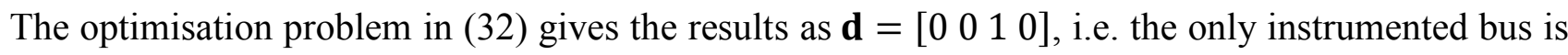
the I220. Hence, the minimum number of measuring devices to obtain a full observability is 12 ; they are detailed in Table 4 and shown in Fig. 4(a).

Table 4 Comparative measuring system for the proposed method

\begin{tabular}{ccc}
\hline Measuring device & Ref. [20] & Proposed \\
\hline Nodal voltage & 12 & 3 \\
Line current & 15 & 9 \\
Total & 27 & 12 \\
\hline
\end{tabular}

This represents a significant saving in the instrumented resources with respect to the case study reported in [20] where the harmonic state was obtained using 27 measuring devices. It represents a reduction of $55 \%$ in the number of measuring devices. 
According to the proposed methodology, voltage and current waveforms have been sampled during half period. The added noise in the measurements has a standard deviation of 0.3 , the fundamental frequency is $f=50 \mathrm{~Hz}$ and the sample period is $T_{\mathrm{S}}=39.0625 \mu$ s, i.e. 256 samples per half period. The filter based on FFT has been applied to mitigate the noise in the measurements during a half period. For illustrative purposes, Fig. 5(a) shows the sampled waveform of the noisy sending end current in line I220-T220 and its corresponding filtered waveform. The noise has been mitigated from 0.3 to 0.03 .

An unbalanced harmonic source, defined in Table 2 and connected at the bus T220, is assumed. The proposed methodology does not need knowledge of the harmonic injection in this bus. By applying the proposed TDHSE to the system shown in Fig. 4(a), the harmonic content is obtained. For illustrative purpose, Fig. 5(b) shows the unbalanced harmonic spectra for the non-monitored branch current I220-T220 line. The non-monitored branch current I220-T220 line has been estimated using (21). The harmonic content of this branch current is compared against those obtained by the SimPowerSystems toolbox of Simulink ${ }^{\circledR}$, taken as the actual values, against the obtained with the method reported in [20]. Good agreement between responses is observed, which verifies the accuracy of the proposed method for HSE. Table 5 shows the resulting error on the THD evaluation. The THD obtained with the proposed methodology is compared against the THD obtained by the method reported in [20] and against the actual power system response. The absolute error of THD is computed. The maximum difference between the actual and the proposed method is 0.0625 .

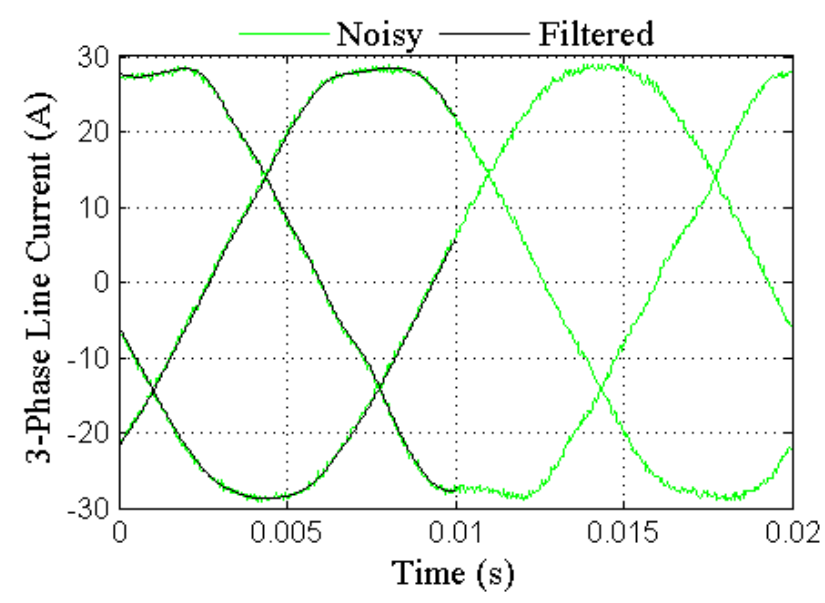

$a$ 


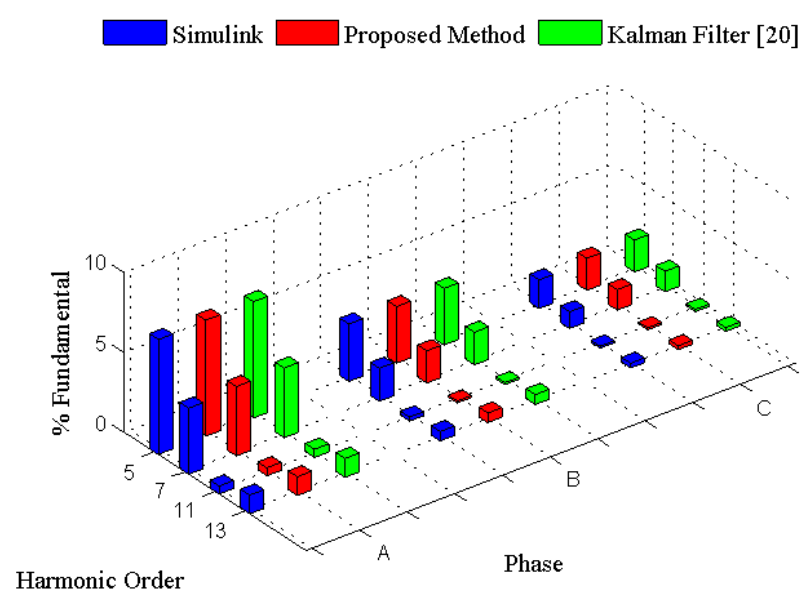

$b$

Fig. 5. I220-T220 line harmonic current estimation

$a$ Half sampled cycle of noisy and filtered measurements of the sending end current waveforms corresponding to line I220$\mathrm{T} 220$

$b$ Comparative harmonic spectra of proposed method against the actual power network response from Simulink ${ }^{\circledR}$ and KF method reported in [20]

Table 5 Comparative THD absolute error for the proposed method

\begin{tabular}{|c|c|c|c|c|c|}
\hline Phase & $\begin{array}{c}\text { Actual } \\
\text { Simulink }{ }^{\circledR}\end{array}$ & $\begin{array}{l}\text { Proposed } \\
\text { Method }\end{array}$ & $\begin{array}{c}\text { KF Method } \\
\text { Ref. [20] }\end{array}$ & $\begin{array}{c}\text { Error } \\
\text { (Proposed } \\
\text { Method) }\end{array}$ & $\begin{array}{c}\text { Error } \\
\text { (KF Method } \\
\text { Ref. [20]) }\end{array}$ \\
\hline A & 8.3695 & 8.4161 & 8.4225 & 0.0466 & 0.0530 \\
\hline B & 4.1848 & 4.2466 & 4.2500 & 0.0619 & 0.0652 \\
\hline $\mathrm{C}$ & 2.0924 & 2.1549 & 2.1554 & 0.0625 & 0.0630 \\
\hline
\end{tabular}

\subsection{Case study 2: Unbalance condition under critically-determined condition}

The purpose of this case study is to show the performance of the proposed method under unbalanced operation conditions. To conduct TDHSE analysis under these conditions, the method has been extended to represent three-phase networks. The modified IEEE 14-bus test system shown in Fig. 4(b) has been selected. The zero sequence parameters of Table 3 are used. The nonlinear load has been disconnected from phase C to introduce unbalance in the power system. For illustrative purposes, Fig. 6 shows the current waveforms in the unbalanced nonlinear load and the resulting voltage waveform at bus 4. Please notice from Fig. 6(a) that the firing angles are approximately 90 degrees. As a result of the unbalanced nonlinear load, the voltage waveforms at bus 4 are highly distorted, as shown in Fig. 6(b). 


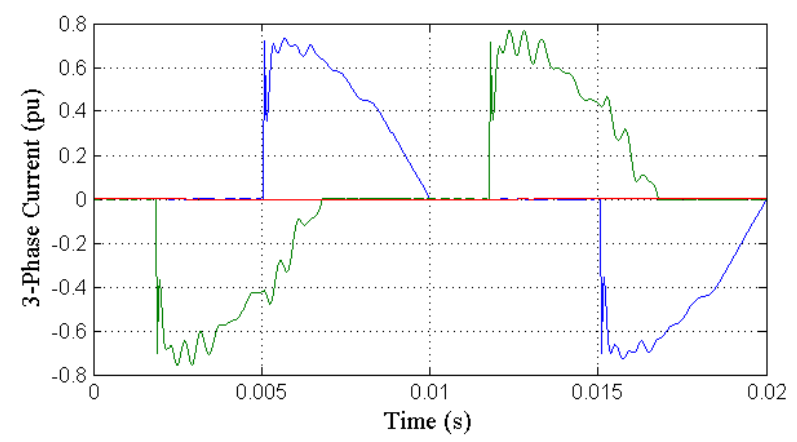

$a$

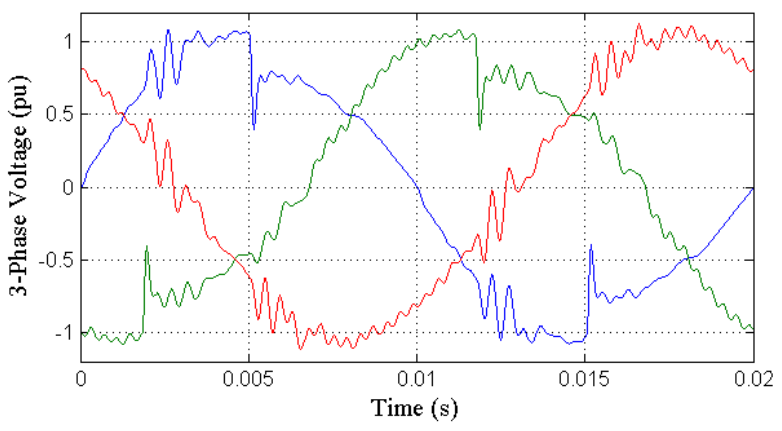

$b$

Fig. 6. Effects of the unbalanced nonlinear load

$a$ Distorted three-phase current waveforms in unbalanced nonlinear load connected at bus 4 .

$b$ Distorted three-phase voltage waveforms at bus 4.

The conducted case study is divided into three parts: First, the filter based on FFT is analysed. Second, the measuring process uses the minimum number of measuring devices, i.e. the critically-determined condition. Finally, two measuring devices are added to obtain an over-determined condition.

4.2.1 Filter based on FFT: The measurements taken from the measuring devices have been contaminated by adding $1 \%$ of random noise. This noise generates different inaccuracies for each harmonic. For this case study, $f=50 \mathrm{~Hz}$ and $T_{\mathrm{S}}=39.0625 \mu$ s. For illustrative purposes, the resulting harmonic content for noisy measurement in phase $\mathrm{A}$ at bus voltage 2 and the generated inaccuracies in each harmonic of the interest for this measurement are given in Table 6 . The maximum absolute error between the actual and the noisy data is 0.0649 present in harmonic 11 . However, the maximum relative error is $6.58 \%$ present in harmonic 21 which is the lowest level. 
Table 6 Inaccuracies generated owing to $1 \%$ of noise

\begin{tabular}{ccccc}
\hline $\begin{array}{c}\text { Harmonic } \\
\text { order }\end{array}$ & $\begin{array}{c}\text { Actual } \\
(\%)\end{array}$ & $\begin{array}{c}\text { Noisy } \\
(\%)\end{array}$ & $\begin{array}{c}\text { Absolute } \\
\text { Error }\end{array}$ & $\begin{array}{c}\text { Relative } \\
\text { Error }(\%)\end{array}$ \\
\hline 3 & 6.6331 & 6.6757 & 0.0426 & 0.6421 \\
5 & 1.8952 & 1.9045 & 0.0093 & 0.4886 \\
7 & 2.2124 & 2.2587 & 0.0463 & 2.0918 \\
9 & 3.2155 & 3.1898 & 0.0257 & 0.7983 \\
11 & 4.5389 & 4.6038 & 0.0649 & 1.4295 \\
13 & 2.2589 & 2.2135 & 0.0454 & 2.0091 \\
15 & 2.0706 & 2.1181 & 0.0475 & 2.2938 \\
17 & 1.7310 & 1.7664 & 0.0354 & 2.0433 \\
19 & 1.3003 & 1.3336 & 0.0333 & 2.5633 \\
21 & 0.5945 & 0.6336 & 0.0392 & 6.5879 \\
23 & 0.7692 & 0.8126 & 0.0435 & 5.6508 \\
25 & 0.7157 & 0.7080 & 0.0077 & 1.0755 \\
\hline
\end{tabular}

To select the harmonics of interest, the filter based on FFT is used. For illustrative purposes, Fig. 7(a) shows the contaminated three-phase voltage waveforms at bus 2 and their respective filtered waveforms. The contaminated three-phase sending end line current waveforms in line 2-3 as well as their respective filtered waveforms are shown in Fig. $7(b)$. The noise has been mitigated from $1 \%$ to $0.2 \%$. Filtered waveforms contain only the harmonics of interest, which are altered by the added noise. In order to exploit the half-wave symmetry of the waveforms, only the half period of the waveforms have been sampled and filtered.

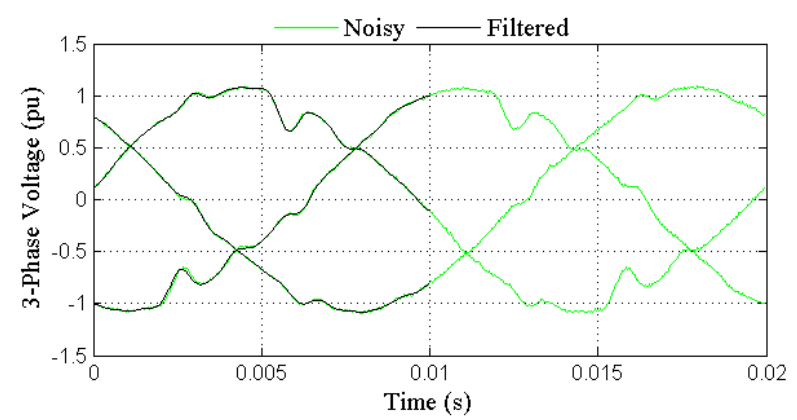

(a) 


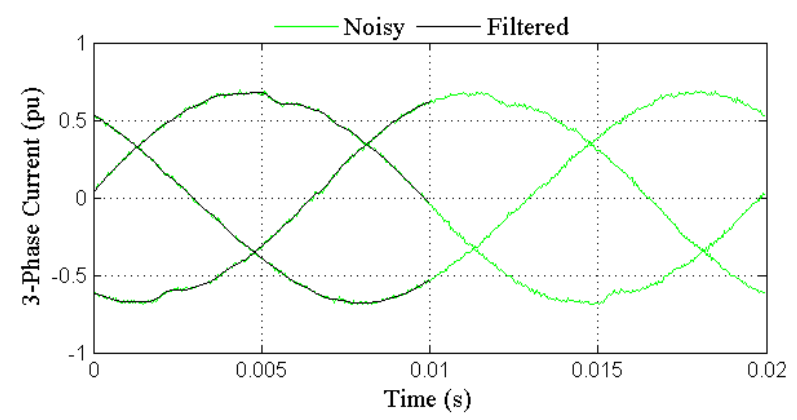

(b)

Fig. 7. Noisy and filtered measurements

$a$ Three-phase voltage waveforms at bus 2 .

$b$ Three-phase sending end current waveforms in line 2-3.

4.2.2 Critically-determined condition: The minimal number of measuring devices that forms the criticallydetermined condition is shown in Fig. 4(b) as green set. The placement is such that the observability is full. This condition forms the matrix $\mathbf{H}_{\mathrm{c}} \in \mathbb{R}^{57 \times 57}$ defined in (33).

$$
\mathbf{H}_{\mathrm{c}}=\left[\begin{array}{ccc}
\mathbf{H}_{\mathbf{1}} & \mathbf{0} \in \mathbb{R}^{21 \times 18} & \mathbf{0} \in \mathbb{R}^{21 \times 18} \\
\mathbf{0} \in \mathbb{R}^{18 \times 21} & \mathbf{H}_{\mathbf{2}} & \mathbf{0} \in \mathbb{R}^{18 \times 18} \\
\mathbf{0} \in \mathbb{R}^{18 \times 21} & \mathbf{0} \in \mathbb{R}^{18 \times 18} & \mathbf{H}_{\mathbf{3}}
\end{array}\right]
$$

where:

$$
\begin{aligned}
& \mathbf{H}_{\mathbf{1}}=\left[\begin{array}{ccccccc}
\mathbf{I} & \mathbf{0} & \mathbf{0} & \mathbf{0} & \mathbf{0} & \mathbf{0} & \mathbf{0} \\
\mathbf{0} & \mathbf{I} & \mathbf{0} & \mathbf{0} & \mathbf{0} & \mathbf{0} & \mathbf{0} \\
\mathbf{0} & \mathbf{0} & \mathbf{I} & \mathbf{0} & \mathbf{0} & \mathbf{0} & \mathbf{0} \\
\mathbf{c} \mathbf{1}_{1-2} & \mathbf{c 2} \mathbf{2}_{1-2} & \mathbf{c} \mathbf{3}_{1-2} & -\mathbf{I} & \mathbf{0} & \mathbf{0} & \mathbf{0} \\
\mathbf{c} \mathbf{1}_{2-3} & \mathbf{c 2} \mathbf{2}_{2-3} & \mathbf{c} 3_{2-3} & \mathbf{0} & -\mathbf{I} & \mathbf{0} & \mathbf{0} \\
\mathbf{c 1}_{2-4} & \mathbf{c 2} & \mathbf{c} 3_{2-4} & \mathbf{0} & \mathbf{0} & -\mathbf{I} & \mathbf{0} \\
\mathbf{c 1}_{2-5} & \mathbf{c 2} 2_{2-5} & \mathbf{c} 3_{2-5} & \mathbf{0} & \mathbf{0} & \mathbf{0} & -\mathbf{I}
\end{array}\right]
\end{aligned}
$$

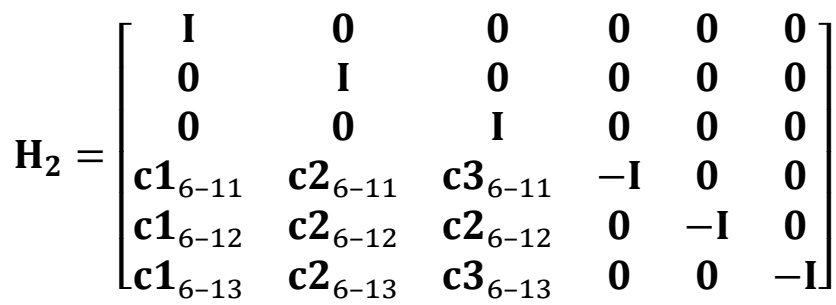

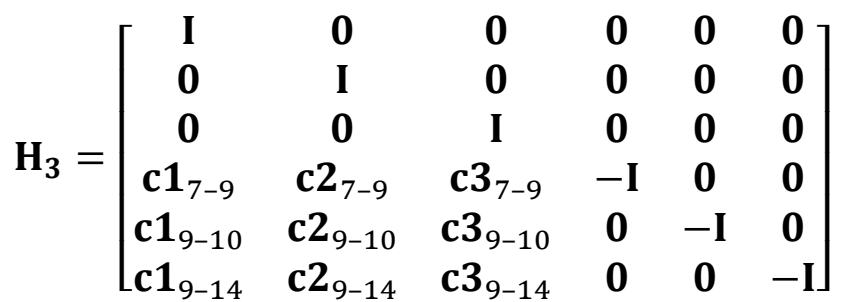

Please note that matrices $\mathbf{0}$ and $\mathbf{I} \in \mathbb{R}^{3 \times 3}$ are the zero and identity matrices, respectively. 
Using (33) in (5) in the proposed methodology, the TDHSE is evaluated. For comparative purposes, the harmonic state at unmonitored bus 5 is analysed. In this case, the power system is modelled with a magnetically coupled three-phase network. Fig. 8 shows the HSE per phase. From Fig. 8 it can be observed that the absolute error is small, e.g. the maximum absolute error in phases $\mathrm{A}, \mathrm{B}$, and $\mathrm{C}$ are $0.57,0.58$ and $0.59 \%$, respectively. In addition, Fig. 8(c) presents harmonic content due to magnetic coupling with phases $\mathrm{A}$ and $\mathrm{B}$. Although the nonlinear load in phases $\mathrm{A}$ and $\mathrm{B}$ is equal, the harmonic content in phases $\mathrm{A}$ and $\mathrm{B}$, shown in Figs. 8(a) and 8(b), respectively, is different due to magnetic coupling. For illustrative purposes, the critically estimated error in phase A is given in detail in Table 7. In order to consider the magnitude of the harmonic, the relative error is used. The maximum relative error is $25.4 \%$ for 19 -th harmonic. The average relative error is $11.1 \%$. The TDHSE accuracy can be improved using the over-determined estimation as will be presented in the next subsection.

4.2.3 Over-determined condition: Two measuring devices have been added at bus 4, as shown in Fig. 4(b). The additional measuring devices are the measurements of the receiving end current in line 4-5 and the sending voltage at bus 4 , marked as the red set. The over-determined condition forms the matrix $\mathbf{H}_{\mathrm{o}} \in$ $\mathbb{R}^{63 \times 57}$ given in (34).

$$
\mathbf{H}_{\mathrm{o}}=\left[\begin{array}{ccc}
\mathbf{H}_{\mathbf{1}} & \mathbf{0} \in \mathbb{R}^{21 \times 18} & \mathbf{0} \in \mathbb{R}^{21 \times 18} \\
\mathbf{0} \in \mathbb{R}^{18 \times 21} & \mathbf{H}_{2} & \mathbf{0} \in \mathbb{R}^{18 \times 18} \\
\mathbf{0} \in \mathbb{R}^{18 \times 21} & \mathbf{0} \in \mathbb{R}^{18 \times 18} & \mathbf{H}_{3} \\
\mathbf{H}_{\mathbf{4}} & \mathbf{0} \in \mathbb{R}^{6 \times 18} & \mathbf{0} \in \mathbb{R}^{6 \times 18}
\end{array}\right]
$$

where:

$$
\mathbf{H}_{\mathbf{4}}=\left[\begin{array}{ccccccc}
\mathbf{0} & \mathbf{0} & \mathbf{0} & \mathbf{0} & \mathbf{0} & \mathbf{I} & \mathbf{0} \\
\mathbf{c 1} \mathbf{1}_{4-5} & \mathbf{c 2} \mathbf{2}_{4-5} & \mathbf{c 3} \mathbf{3}_{4-5} & \mathbf{0} & \mathbf{0} & \mathbf{0} & -\mathbf{I}
\end{array}\right]
$$

For comparative purpose, the harmonic content for the actual and the estimated harmonic, named over-estimated, at unmonitored bus 5 is also shown in Fig. 8. Please observe that the absolute error has notoriously been reduced, e.g. the maximum differences for phases A, B, and C has been reduced from 0.57 to 0.22 , from 0.58 to 0.21 , and from 0.59 to $0.17 \%$, respectively. Table 7 gives in detail the estimated error for the critical and over HSE conditions in phase A. The average relative error in phase A has been reduced from 10.4 to $2.85 \%$. The maximum error is $8.8 \%$ and corresponds to $21-t h$ harmonic which has the smallest magnitude. 


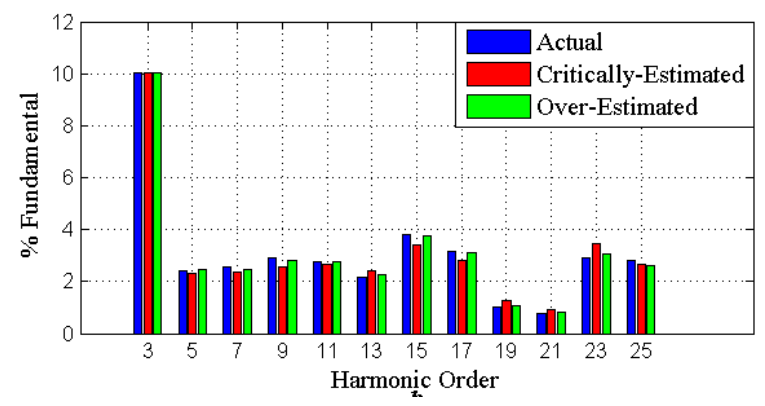

(a)

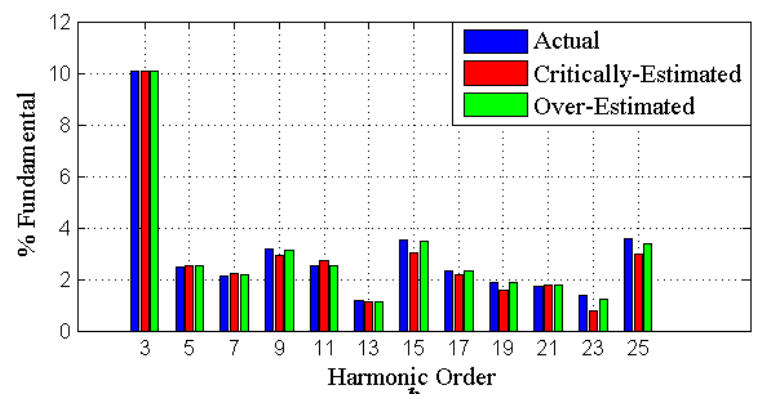

(b)

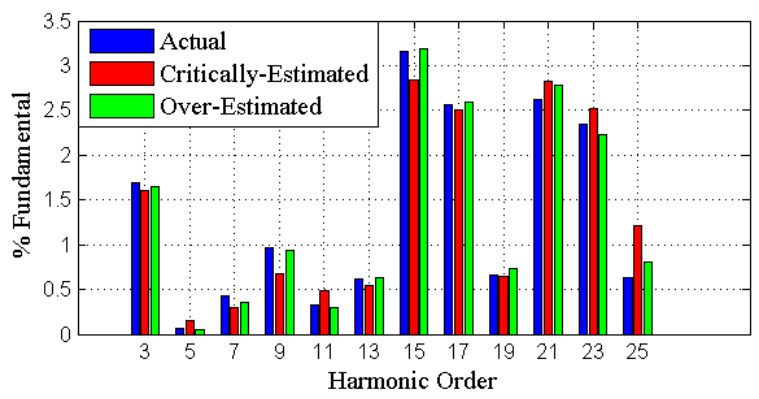

(c)

Fig. 8. Harmonic state estimation for bus 5

$a$ Spectra for phase A.

$b$ Spectra for phase B.

$c$ Spectra for phase C.

Table 7 Estimate error for HSE at unmonitored phase A in bus voltage 5 (\%)

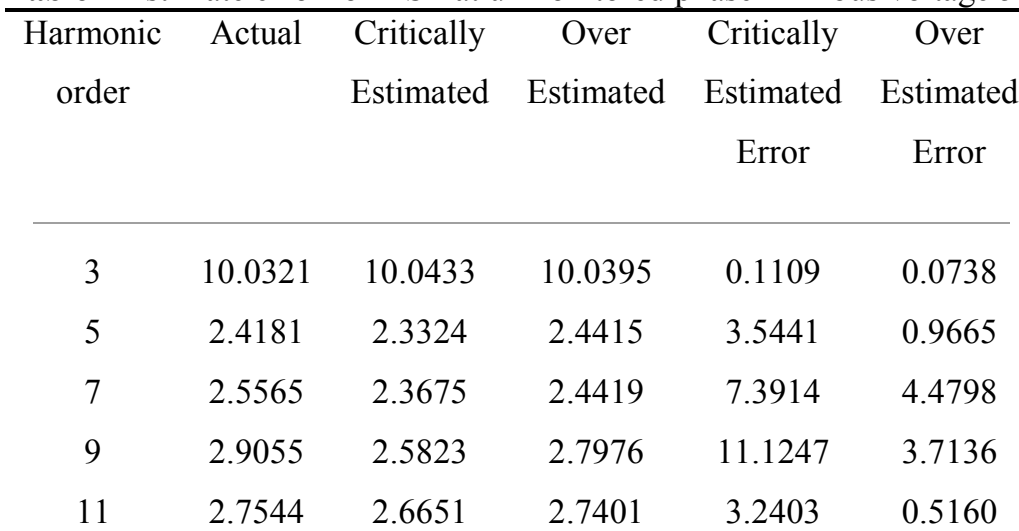




\begin{tabular}{llllll}
13 & 2.1441 & 2.4360 & 2.2839 & 13.6137 & 6.5203 \\
15 & 3.8259 & 3.3867 & 3.7616 & 11.4783 & 1.6802 \\
17 & 3.1735 & 2.8124 & 3.0922 & 11.3795 & 2.5601 \\
19 & 1.0202 & 1.2802 & 1.0670 & 25.4867 & 4.5869 \\
21 & 0.7590 & 0.9147 & 0.8265 & 20.5112 & 8.8904 \\
23 & 2.9000 & 3.4708 & 3.0584 & 19.6796 & 5.4605 \\
25 & 2.8308 & 2.6638 & 2.6097 & 5.9003 & 7.8124 \\
\hline
\end{tabular}

4.2.4 Under-determined condition: The proposed method uses decoupled equations for state variables. Thus, the under-determined condition can be changed to properly determined condition but partially observable if unobservable state variables are defined. For instance, if the current measurements in line 2-4 for the IEEE 14 bus test system are not available, the resulting matrix is $\mathbf{H} \in \mathbb{R}^{54 \times 57}$, i.e. the under-determined condition. If the bus voltage 4 is defined as unobservable, the measurement matrix changes to properly determined condition, i.e. $\mathbf{H} \in \mathbb{R}^{54 \times 54}$. The solution for the rest of buses is similar to the critically determined condition, except for the bus 4 that has been defined unobservable.

4.2.5 Four-wire circuit case study: The load connected at bus 13 is modelled as a 4-wire circuit. The estimated load currents as well as the estimated neutral current are shown in Fig. 9(a). The respective harmonic components are shown in Fig. 9(b); as expected, the triplen harmonic currents $(3,9,15$, and 21) are of considerable magnitude. Due to the existing unbalance operation condition, harmonics of different order are produced.

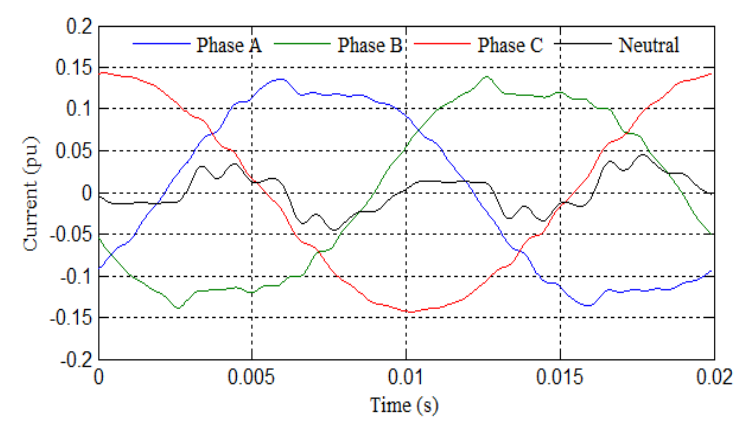

$(a$

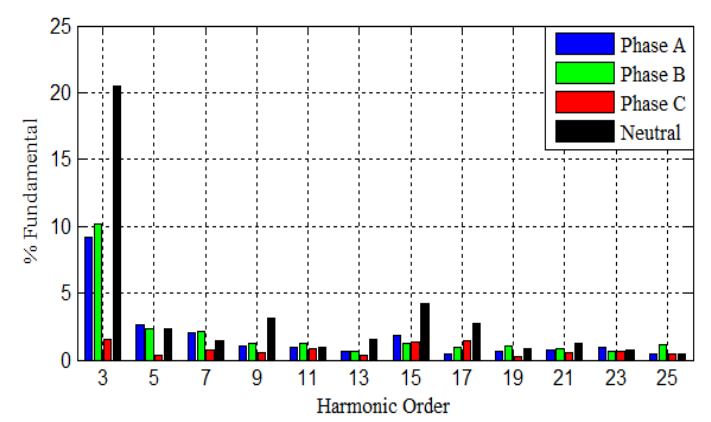

(b 
Fig. 9 Estimated phase and neutral load currents at unmonitored bus 13.

$a$ Waveforms.

$b$ Harmonic content.

\section{Conclusions}

A time domain methodology for harmonic state estimation assessment (TDHSE) has been proposed. It can be applied in both balanced and unbalanced three-phase power systems.

A method to obtain the optimal number of measuring devices and their appropriate placement to evaluate the HSE in the time domain has been proposed. This can significantly reduce the cost of instrumentation.

By taking advantage of the half-wave symmetry property in the waveforms of voltage and current, the execution time to evaluate the HSE in the time domain has been reduced by approximately $50 \%$ of the conventional solution process as only half period of the waveforms is sampled and processed.

The results indicate that using the critically-determined condition, the estimation error can be acceptable for harmonics whose magnitude is larger than the noise level.

Besides, the results suggest that the accuracy of the HSE, including harmonics whose magnitude is small compared to the noise level, can be improved if the over-determined condition is used.

The proposed methodology does not require of an accurate previous knowledge of the harmonic sources nor a procedure for the determination of precise initial state conditions.

\section{Acknowledgments}

The authors gratefully acknowledge to the Universidad Michoacana de San Nicolás de Hidalgo through the Facultad de Ingeniería Eléctrica, División de Estudios de Posgrado (FIE-DEP) Morelia, México, for the facilities granted to carry out this investigation. The first author wishes to thank the study leave granted by the Instituto Tecnológico de Morelia and to the Consejo Nacional de Ciencia y Tecnología of México (CONACYT) for finance assistance for his doctoral studies at the FIE-DEP. The second author acknowledges financial support from CONACYT for this investigation.

\section{References}

[1] Dugan, R.C., Santoso, S., McGranaghan, M.F., Wayne Beaty, H.: 'Electrical power systems quality' (McGrawHill, 2002, 2nd edn.)

[2] Arrillaga, J., Watson, N., Chen, S.: 'Power system quality assessment' (John Wiley \& Sons, 2000, 2nd edn.)

[3] Watson, N.R., 'Power quality state estimation', Euro. Trans. Elec. Power, 2010, 20, pp. 19-33

[4] Rakpenthai, C., Uatrongjit, S., Watson N.R., Premrudeepreechacharn, S.: 'On harmonic state estimation of power system with uncertain network parameters', IEEE Trans. Power Syst., 2013, 28, (4), pp. 4829-4838 
[5] Abbas Ketabi, A., Reza, M., Nosratabadi, S.: 'Power quality meters placement using seeker optimization algorithm for harmonic state estimation', Int. J. Elect. Power Energy Syst., 2012, 1, (43), pp. 141-149

[6] Rad, M., Mokhtari, H., Karimi, H.: 'A new algorithm for optimal measurements placement, observability analysis and harmonic state estimation in power systems', Pow. Elect. Drive Sys. \& Tech. Conf., Theran, Iran, Feb 2013, pp. $1-6$

[7] Nguyen, H., Yang, J., Choi, S.: 'On harmonic state estimation and the evaluation of harmonic power contribution from sources', Power and Ener. Soc. Gen. Meet., Minnesota, USA, Jul. 2010, pp. 1-6

[8] Almeida, C., Kagan, N.: 'Harmonic state estimation through optimal monitoring systems', IEEE Trans. Smart Grid, 2013, 4, (1), pp. 467-478

[9] Saxena, D., Bhaumik, S., Singh, S.: 'Identification of multiple harmonic sources in power system using optimally placed voltage measurement devices', IEEE Trans. Ind. Elect., 2014, 61, (5), pp. 2483-492

[10] Eldery, M.A., El-Saadany, E.F., Salama, M.M.A., Vannelli, A.: 'A novel power quality monitoring allocation algorithm', IEEE Trans. Power Deliv., 2006, 21, (2), pp. 768-777

[11] Madtharad, C., Premrudeepreechacharn, S., Watson, N.R., Saeng-Udom, R.: 'An optimal measurement placement method for power system harmonic state estimation', IEEE Trans. Power Deliv., 2005, 20, (2), pp. 1514-1521

[12] Wen, H., Zhang, J., Meng, Z., Guo, S., Li, F., Yang, Y.: 'Harmonic estimation using symmetrical interpolation FFT based on triangular self-convolution window', IEEE Trans. Ind. Inf., 2015, 11, (1), pp. 16-26

[13] Lázaro, J., Miñambres, J., Zorrozua, M.: 'Selective estimation of harmonic components in noisy electrical signals for protective relaying purposes', Int. J. Elect. Power Energy Syst., 2014, 1, (56), pp. 140-146

[14] Kumar, P., Subudhi, B.: 'Ensemble-Kalman-filter-based power system harmonic estimation', IEEE Trans. Inst. \& Meas., 2012, 61, (12), pp. 3216-3224

[15] Arrillaga, J., Watson, N., 'Power system harmonics', (John Wiley \& Sons, 2003, 2nd edn.)

[16] Watson, N., Arrillaga, J., 'Power systems electromagnetic transients simulation', (IET Power and Energy Series 39, 2003, 1st edn.)

[17] Bharata, M., Sagar, K., Mohanta, D.: 'A multifunctional real-time power quality monitoring system using Stockwell transform', IET Sci. Meas. Technol., 2014, 8, (4), pp. 155-169

[18] Siddiqui, R., Grosvenor, R., Pricket, P.: 'dsPIC-based advanced data acquisition system for monitoring, control and security applications', Proc. Int. Conf. on Applied Sciences \& Technology, Islamabad, Pakistan, Jan 2015, pp. 293-298

[19] 'GPS synchronization architecture for data acquisition devices', http:/www.ni.com/white-paper/7023/en/, accessed February 2017

[20] Medina, A., Cisneros-Magaña, R.: 'Time-domain harmonic state estimation based on the Kalman filter Poincaré map and extrapolation to the limit cycle', IET Gener. Transm. Distrib., 2012, 6, (12), pp. 1209-1217

[21] Semlyen, A., Medina, A.: 'Computation of the periodic steady state in systems with nonlinear components using a hybrid time and frequency domain methodology', IEEE Trans. Power Syst., 1995, 10, (3), pp. 1498-1504 
[22] Cisneros-Magaña, R., Medina, A., Segundo-Ramírez, J.: 'Efficient time domain power quality state estimation using the enhanced numerical differentiation Newton type method', Int. J. Elect. Power Energy Syst., 2014, 1, (63), pp. 414-422

[23] Molina-Moreno, I., Medina, A., Cisneros-Magaña, R.: 'Experimental time domain harmonic state estimation using partial measurements', North American Power Symposium, Pullman, USA, Sep 2014, pp. 1-6

[24] Molina-Moreno, I., Medina, A., Cisneros-Magaña, R.: 'Time domain harmonic state estimation using filtered measurements based on Fourier transform', North American Power Symposium, Charlotte, USA, Oct 2015, pp. 1-6

[25] Schweppe, F.C., Handschin, E.J.: 'Static state estimation in electric power systems', Proc. IEEE, 1974, 62, (7), pp. $972-982$

[26] Monticelli, A.: 'Electric power system state estimation', Proc. IEEE, 2000, 88, (2), pp. 262-282

[27] Weng, Y., Ilic, M.D., Li, Q., Negi, R.: 'Convexification of bad data and topology error detection and identification problems in AC electric power systems', IET Gener. Transm. Distrib., 2015, 9, (16), pp. 2760-2767

[28] Jeffrey, A.: 'Matrix operations for engineers and scientists: an essential guide in linear algebra' (Springer, 2010, 1st edn.)

[29] Burden, R., Fires, J.: 'Numerical analysis' (CENGAGE Learning, 2011, 9th edn.)

[30] Grainger, J., Stevenson, W.; 'Power system analysis' (McGraw-Hill, 1994, 1st edn.)

[31] Arrillaga, J., Arnold, C.P.: 'Computer analysis of power systems', (John Wiley \& Sons, 1994.)

[32] Task Force on harmonics modeling and simulation.: 'Modeling and simulation of the propagation of harmonics in electric power networks. Part I concepts, models and simulation techniques', IEEE Trans. Power Deliv., 1996, 11, (1), pp. $452-465$

[33] Acha, E., Madrigal, M.: 'Power systems harmonics computer modelling and analysis', (John Wiley \& Sons, 2001.)

[34] Korres, G.N., Manousakis, M.N., Xygkis, T.C., Lofberg, J.: ' Optimal phasor measurement unit placement for numerical observability in the presence of conventional measurements using semi definite programming', IET Gener. Transm. Distrib., 2015, 9, (15), pp. 2427-2436

[35] Koutsoukis, N.C., Manousakis, N.M., Georgilakis, P.S., Korres, G.N.: 'Numerical observability method for optimal phasor measurement units placement using recursive Tabu search method', IET Gener. Transm. Distrib., 2013, 7, (3), pp. 347-356

[36] Rashidi, F., Abiri, E., Niknam, T., Reza Salehi, M.: 'Optimal placement of PMUs with limited number of channels for complete topological observability of power systems under various contingencies', Int. J. Elect. Power Energy Syst., 2015, 1, (67), pp. 125-137

[37] Saha, B.K., Sinha, A.K., Pradhan, A.K.: 'An optimal PMU placement technique for power system observability', Int. J. Elect. Power Energy Syst., 2012, 1, (42), pp. 71-77

[38] Mahari, A., Seyedi, H.: 'Optimal PMU placement for power system observability using BICA, considering measurement redundancy', Electr. Power Syst. Res., 2013, 103, (1), pp. 78-85 
[39] Müller, H., Castro, C.: 'Genetic algorithm- based phasor measurement unit placement method considering observability and security criteria', IET Gener. Transm. Distrib., 2016, 10, (1), pp. 270-280

[40] Gou, B.: 'Optimal placement of PMUs by integer linear programming', IEEE Trans. Power Syst., 2008, 23, (3), pp. 1-2

[41] Suresh, K.: 'Electric circuits and networks', (Pearson Education, 2009, 1st edn.)

[42] Rashid, M.H.: 'Power electronics: circuits, devices, and applications', (Pearson Education, 2014, 4th edn.)

[43] 'University of Washington, Electrical Engineering, Power Systems Test Case Archive', http://www.ee.washington.edu/research/pstca, accessed: February 2017

[44] Kundur, P.: 'Power system stability and control', (McGraw-Hill, 1994, 1st edn.) 